\title{
Fonction des équivalences dans un dictionnaire bilingue
}

\section{Martine Schuwer}

\section{(2) OpenEdition}

Journals

Édition électronique

URL : http://journals.openedition.org/asp/4239

DOI : 10.4000/asp.4239

ISSN : 2108-6354

Éditeur

Groupe d'étude et de recherche en anglais de spécialité

Édition imprimée

Date de publication : 1 février 1994

Pagination : 83-94

ISSN : 1246-8185

Référence électronique

Martine Schuwer, «Fonction des équivalences dans un dictionnaire bilingue », ASp [En ligne], 3| 1994, mis en ligne le 20 février 2014, consulté le 19 avril 2019. URL : http://journals.openedition.org/ asp/4239 ; DOI : 10.4000/asp.4239

Ce document a été généré automatiquement le 19 avril 2019

Tous droits réservés 


\title{
Fonction des équivalences dans un dictionnaire bilingue
}

\author{
Martine Schuwer
}

1 Dans un dictionnaire bilingue, on conçoit bien l'objectif explicite du lexicographe qui propose, pour un terme, plusieurs équivalences dans la langue cible. Il rend ainsi compte de la non-isomorphie bien établie des champs sémantiques. Mais au-delà de ces informations, quelle fonction ces équivalences jouent-elles pour le lecteur?

2 Tout d'abord, ces équivalences ne seraient pas à considérer isolément, mais dans leur ensemble; c'est ce qu'elles partagent - leur «fonds commun sémantique» - qui constituerait, en réalité, le champ sémantique du terme source et que le lexicographe aurait ainsi cherché, implicitement, à reconstruire. On pourrait dire qu'en d'autres termes, ces équivalences correspondraient à un essai de définition translinguistique du terme source.

3 Force est de constater, par ailleurs, que les équivalences proposées sont toujours incomplètes (tout comme une définition ne rend pas compte de la totalité des nuances de sens d'un mot). Le dictionnaire s'avère donc toujours en deçà de la demande du traducteur.

4 Pour quelle raison, et comment serait-il possible de remédier à ces manques?

5 C'est parce qu'il y a, à la source, inadéquation entre l'objectivité désirée du dictionnaire et la subjectivité essentielle de l'activité de langage. Le dictionnaire est en effet un répertoire de signes objectivement identifiés. Le signe est certes porteur de sens, mais sa signification ne se réalise que dans l'acte d'énonciation. Le mot est en fait la réalisation ponctuelle de la notion dans une situation d'énonciation donnée, et évidemment imprévisible.

6 Ainsi, puisque le lexicographe se trouve dans l'impossibilité absolue de faire la liste de toutes les équivalences possibles (et appropriées) d'un mot, il lui faut changer d'objectif : ne plus chercher à mettre en équivalence le mot, mais son « domaine notionnel », ce qui permettra à l'utilisateur de « re-créer du sens », qui est le propre de l'activité de langage. 
mentant la méthode de travail des linguistes, Roman Jakobson notait que « quand on se sert trop longtemps d'une technique, on finit par croire que c'est la procédure normale » (Jacobson 1963 : chapitre 1). effet, lorsqu'on essaie de découvrir selon quels principes un dictionnaire bilingue général est conçu, et quel usage on est censé en faire, on peut se demander si les règles qui président à sa macro/microstructure ne dérivent pas de postulats aux fondements insuffisamment étayés.

quels présupposés la conception repose-t-elle? Au stade de l'élaboration tout d'abord ne prend-on pas pour acquis, par exemple, le fait qu'un article comprendra un certain nombre de subdivisions sémantiques (établie selon quels critères?), au stade de l'utilisation ensuite, que l'utilisateur «fera bon usage» (lequel et comment?) des informations mises à sa disposition?

10 C'est par le biais de l'analyse de la fonction des équivalences dans un dictionnaire bilingue que l'on va tenter de comprendre dans quelle mesure ces présupposés entraînent des dysfonctionnements d'origine structurelle.

\section{Le cadre de travail}

11 Après avoir réalisé, sur informatique, la version anglais-français du Dictionnaire bilingue de l'environnement (Collin \& Schuwer 1992), j'avais procédé au retournement informatique des données afin d'en obtenir la version français-anglais. Cette réorganisation des données avait fait apparaître un certain nombre d'aberrations (Schuwer 1993) qui étaient de deux ordres : morphosyntaxique, et sémantique.

Pour éliminer celles d'origine morphosyntaxique, il avait été proposé d'insérer des balises appropriées dans la version anglais-français. Même si elles ne pouvaient faire l'objet d'une macrocommande - chaque unité lexicale devant être traitée individuellement ces aberrations étaient néanmoins prévisibles.

13 Malgré ces améliorations, la nouvelle version comportait encore bon nombre d'articles au contenu insatisfaisant, le plus souvent parce qu'il était incomplet.

14 Ainsi, dans la version retournée «brute » - c'est-à-dire telle qu'elle apparaissait après le retournement automatique - on pouvait trouver par exemple :

sol nm ground

terrain nm ground

terre nf ground

ce qui était finalement prévisible puisque pour ground, dans la $1^{\text {re }}$ version, on avait donné terre, terrain, sol.

15 Pour améliorer la $2^{\mathrm{e}}$ version, le lexicographe doit alors rajouter les équivalences manquantes soil, land, earth, voire country.

On comprend aisément pourquoi, à l'issue du retournement automatique, les équivalences sont incomplètes : le lexicographe avait programmé un simple changement de place des rubriques ; il obtient ce qu'il avait demandé !

En conclusion de cette recherche, on avait commencé à s'interroger sur la fonction des équivalences dans un dictionnaire bilingue ; on avait suggéré que les équivalences proposées ne sont pas une suite de termes à considérer individuellement, mais que pour « saisir » le sens de ground, il faut les appréhender dans leur ensemble, que c'est ce que 
ces équivalences ont en commun qui constituerait le champ sémantique du terme source, et qui représenterait le "fonds commun sémantique» des équivalences. Ainsi le lexicographe, pour chaque lexie, se livrerait-il à la reconstitution, par ajout successif d'équivalences, du champ sémantique du terme source.

\section{Microstructure - subdivisions sémantiques}

18 J'avais souligné le caractère très probablement inconscient de la démarche du lexicographe dans la détermination de ses choix.

19 Mais quelle stratégie met-iI en œuvre? Il est légitime de penser que l'intuition est son guide constant, son unique référence, même si dans un souci d'exactitude, il contrôle parfois - et le plus souvent dans un autre dictionnaire - la justesse de son intuition.

\subsection{Constat et principes}

20 Aucun lexicographe ${ }^{1}$, à notre connaissance, n'a encore dévoilé sa stratégie, ce qui va de pair, à mon sens, avec l'absence totale, dans l'introduction des dictionnaires bilingues, de conseils sur «la meilleure façon d'utiliser ce dictionnaire ». Que le lexicographe n'expose pas sa méthodologie, soit, mais l'utilisation du dictionnaire va-t-elle vraiment de soi ? L'absence d'instructions le laisserait supposer, on pourrait même penser que les innombrables détails sur la macro/microstructure sont là pour compenser ce manque, comme en témoignent ces deux extraits d'introduction de dictionnaires bilingues :

« Tout article complexe est clairement divisé en catégories sémantiques introduites par une indication qui en donne le sens général. De plus les variations de sens à l'intérieur de ces catégories sont soigneusement mises en évidence à l'aide de renseignements supplémentaires précisant chaque nuance. L'utilisation cohérente de ce système d'indications, sous forme de synonymes, de définitions partielles ou de compléments à valeur typique est l'une des caractéristiques essentielles de ce dictionnaire ». (Robert \& Collins 1987)

«Des lettres sont utilisées à l'intérieur de chaque catégorie grammaticale pour marquer un changement de sens. [...]

Au sein des articles, les virgules séparent les traductions ayant le même sens ou le même emploi. Un point virgule séparant deux traductions indique une nuance de sens et est généralement accompagné d'une indication expliquant cette nuance [...] Les synonymes et explications en italiques servent à déterminer le sens du mot traduit $[\ldots]$

Les exemples types d'emploi apparaissant en italique et entre parenthèses indiquent quel genre de combinaisons sont possibles [sic] avec une traduction particulière. » (Harrap's Shorter 1991)

21 Quoiqu'on pense de l'opportunité - pour le lecteur - de ces remarques, il faut souligner qu'un même souci guide ces deux équipes de lexicographes : assurer le lecteur qu'ils ont répertorié et présenté les «nuances » ou « variations » de sens de la façon la plus précise et objectivement compréhensible possible. On peut noter cependant que seul Robert \& Collins introduit la notion de catégorie sémantique là où Harrap's parle de changement de sens.

Il faut maintenant s'interroger sur la division en catégories sémantiques: selon quels critères est-elle établie? 


\subsection{Réalités}

23 À titre d'exemple voici, pour le terme ground, la subdivision comparée des mêmes deux dictionnaires bilingues anglais-français ${ }^{2}$ (tableau 1).

\begin{tabular}{|l|l|}
\hline Robert \& Collins & Harrap's \\
\hline (a) (U) la terre, le sol & (a) sol, terre \\
\hline (b) (U: soil) sol, terre, terrain & (b) (land) terrain \\
\hline $\begin{array}{l}\text { (c) (U) (piece of land) terrain, domaine, terres, territoire, } \\
\text { sol }\end{array}$ & (c) fond (de la mer) \\
\hline (d) (area for special purpose) terrain & (d) (background) fond, champ \\
\hline (e) (gardens) parc & (e) (reason) raison, cause \\
\hline (f) Am, Elec terre, masse & (f) (US Elec) masse, terre \\
\hline (g) (reason: gen: s) motif, raison & (g) grounds (of coffee...) marc \\
\hline (h) (background) fond & $\begin{array}{l}\text { (h) Mus ground note: } \\
\text { fondamental }\end{array}$ \\
\hline
\end{tabular}

24 Notre propos n'est pas d'évaluer les mérites comparés des deux catégorisations, de les justifier ou les critiquer, mais de souligner les différences d'organisation interne d'un article à l'autre : les subdivisions ne sont pas semblables, et on peut conclure que c'est déjà la preuve qu'il n'existe pas de découpage sémantique établi, avéré, attesté.

Ces deux catégorisations sont donc éminemment subjectives, ce qui ne veut pas dire arbitraires, car chaque lexicographe serait tout à fait en mesure de justifier son choix. Il est même probable que si chacun d'entre nous devait construire une telle souscatégorisation sémantique, on obtiendrait plusieurs organisations différentes. Notons cependant que si l'on regroupe toutes ces équivalences, on finit par couvrir le champ sémantique du terme-source, ce qui est, en un sens, rassurant.

\subsection{Essai d'interprétation des différences}

Comment interpréter le caractère variable de ces subdivisions ? On peut imaginer deux types de causes : cela peut être dû soit à l'état de la recherche en sémantique - on n'aurait pas encore réussi à élucider la structure interne objective du champ sémantique considéré -, soit cette structure interne ne s'imposerait pas de façon patente et serait donc laissée à l'interprétation subjective de chaque lexicographe, et donc chaque utilisateur. Les limites du champ sémantique seraient ainsi invariantes, tandis que le découpage interne serait sujet à interprétation subjective. 
Quoi qu'il en soit, la question est bien le champ sémantique, et si on n'arrive pas à apporter de réponse, c'est peut-être la trace d'un autre problème, en amont, qui n'a pas encore été résolu.

\section{3. Équivalences translinguistiques = définition intralinguistique?}

Cette question du champ sémantique évoque celle de la définition dans un dictionnaire unilingue.

Matériellement parlant, les équivalences du dictionnaire bilingue occupent la place de la définition du dictionnaire unilingue. Serait-il donc erroné de penser que les équivalences correspondraient à un essai de définition translinguistique du terme source?

Avant d'examiner cette question, il faut faire le point sur les définitions possibles de la définition.

Alain Rey souligne d'emblée l'ambiguïté du mot car

selon les domaines du savoir, philosophie, logique, sémantique lexicale, lexicologie, terminologie, et à l'intérieur de chaque domaine selon les théories et les pratiques,

l'objectif [de la définition] peut varier énormément. (Rey $1990: 14)^{3}$

Dans le dictionnaire bilingue, qui, traditionnellement, ne comporte pas de définition, il semblerait qu'on n'évite pourtant pas l'ambiguïté fondamentale propre à toute définition de dictionnaire entre signifier et être, entre ce que le mot "signifie », et ce que le référent « est » puisque l'objectif est de dire comment le terme source peut se traduire - c'est-àdire comment il peut «être signifié »- dans la langue cible. Le lexicographe travaillant sur un dictionnaire bilingue se voit donc contraint de se préoccuper d'une part de "constitution" intralinguistique des connaissances, d'autre part de "transmission [translinguistique] de ces connaissances».

D'autres travaux sur la définition ont posé le problème en termes de sémantique du prototype (Kleiber 1990), dont l'objet est l'élaboration d'une théorie du sens linguistique, et plus particulièrement du sens d'un mot, de la détermination des éléments pertinents qui doivent figurer dans sa définition.

Cela ne nous éloigne pas de notre sujet puisque, dans un dictionnaire bilingue, il s'agit de donner la (les) meilleure(s) traduction(s) possible(s) pour un terme source, et que dans un dictionnaire unilingue, l'objectif de la définition correspond à la recherche des traits pertinents et/ou caractéristiques définissant au mieux un mot. G. Kleiber écarte la théorie trop restrictive des "conditions nécessaires et suffisantes" (CNS) comme solution opérante dans l'essai de définition pour aboutir à ce qu'il nomme la «version polysémique du prototype ». Les différents emplois d'un mot dériveraient tous d'un "sens basique ». Cette nouvelle version de la sémantique du prototype permettrait de rendre compte de la polysémie d'une unité lexicale. Certes, le caractère non isomorphe des champs sémantiques des différentes langues a depuis longtemps été démontré, mais on peut aller au-delà de ce constat, car, appliquée à la lexicographie bilingue, cette théorie de Kleiber explique pourquoi les différents « emplois » d'un même mot en langue source se trouvent réalisés en un certain nombre d'équivalences, et qu'il y a un lien entre ces termes de la langue cible. 
35 À la multiplicité des objectifs possibles de la définition s'ajoute ainsi la pluralité des réalisations linguistiques. Le lexicographe auteur d'un dictionnaire bilingue cumule ainsi les obstacles: il doit en premier lieu et implicitement déterminer la "définition » du terme dans la langue source (et on a pu constater que cette question n'est simple qu'en apparence) puis donner les équivalences dans la langue cible.

Cette opération intermédiaire met en évidence le lien de parenté sémantique qui existe entre la définition et les équivalences. Certes, par nature elles diffèrent (la réalisation est intralinguistique ou interlinguistique) mais leur fonction de "correspondance sémantique » est comparable.

\section{4. «It depends on the context »}

37 À ce stade, on a donc déterminé deux interprétations non exclusives de la fonction des équivalences dans un dictionnaire bilingue : d'une part, elles seraient à considérer sous l'angle $\mathrm{du}$ « fond commun sémantique » qu'elles partagent, d'autre part elles joueraient le rôle d'une définition translinguistique.

Confrontons ces interprétations à la réalité de la démarche du traducteur. Force est de constater qu'il va souvent choisir, et à juste titre, de traduire à l'aide d'un terme qui ne figurera même pas parmi les équivalences proposées, et cela n'étonne personne.

Les dictionnaires bilingues sont-ils mal conçus ? Sont-ils incomplets?

Il serait injuste de ne pas rendre compte des énormes progrès réalisés dans ce domaine depuis une vingtaine d'années, le Robert \& Collins ayant véritablement inauguré un nouveau type de dictionnaire bilingue, riche d'indicateurs de domaine et de nombreux exemples montrant que «les traductions ne sont pas fixes, et qu'elles peuvent changer suivant le contexte » (Robert \& Collins $1987: \mathrm{x}$ ). Néanmoins, disposant d'un outil nettement plus performant, les étudiants d'aujourd'hui ne font pas de meilleures traductions que ceux des générations précédentes. L'outil ne fait pas tout, ce type d'outil là en tout cas.

Le discours que les enseignants tiennent aux étudiants est d'ailleurs très révélateur. On ne cesse de leur dire :

- d'apprendre à se servir du dictionnaire (leur apprend-on ? Et comment ?)

- de se méfier du dictionnaire.

- que, dans ce contexte, on traduirait plutôt par tel ou tel mot qui, bien entendu, ne figure pas dans la liste des équivalences proposées.

On tient, en définitive, rarement des propos positifs sur l'utilisation du dictionnaire.bilingue. On va même jusqu'à la déconseiller, car elle s'avère trop fréquemment génératrice d'erreurs chez des utilisateurs inexpérimentés. Et les enseignants sont les témoins quotidiens de l'inadéquation de l'outil et de la tache. Puisqu'on a constaté que le dictionnaire bilingue actuel est plutôt mieux conçu, mais que néanmoins on se heurte toujours aux mêmes difficultés, faudrait-il en conclure qu'il donc inadapté par nature? Et si oui, pourquoi? 


\section{5. « Don't look for the meaning of a word, look for its use! »}

En d'autres termes ${ }^{4}$, on devine intuitivement qu'un dictionnaire deux fois plus « fourni » resterait toujours en deçà de nos besoins, et de son objet. Qu'a-t-il donc comme caractéristique fondamentale qui le rend apparemment si inadapté à remplir la fonction souhaitée?

C'est qu'en réalité, le dictionnaire est un répertoire de signes et que le lexicographe bilingue veut faire correspondre un système de signes à un autre système de signes ${ }^{5}$.

Est «signe » ce que les locuteurs natifs reconnaissent comme « mot » existant dans leur langue, donc ayant un sens. Et Benveniste a bien souligné que le signe «a toujours et seulement valeur générique et conceptuelle. Il n'admet pas de signifié particulier ou occasionnel ». C'est là le cœur du problème.

Le dictionnaire est bien une suite alphabétique de signes répertoriés sous forme d'entrées, unités de base en lexicographie.

Le lexicographe reconnaît tout d'abord le signe comme une unité lexicale porteuse de sens, il l'étudie en lui-même, c'est-à-dire qu'il s'efforce de le définir de la façon la plus objective possible, en dehors de toute situation, de tout contexte susceptible d'en colorer, donc d'en altérer le sens, hors de toute actualisation dans le discours. Ce n'est qu'au stade des exemples qu'il adoptera une perspective différente, disons pragmatique, où il présentera le mot dans une phrase afin d'illustrer ce que dans la pratique, sa définition implicite contenait. Le signe est donc bien porteur de sens, mais ce n'est que dans l'acte d'énonciation que le sens va s'accomplir dans et par une forme spécifique.

Dans le cas de la lexicographie bilingue, ceci explique pour quelle raison, quel que soit le nombre des équivalences citées, le lexicographe ne peut, de par sa perspective de travail - répertorier les signes, les mettre en équivalences - prévoir comment le signe traité s'articulera avec les autres éléments de la phrase au contact desquels il se réalisera dans sa signification ponctuelle, particulière.

Ce n'est donc pas pour des considérations pratiques (manque de place, incompétence du lexicographe...) que l'on ne trouve pas tout dans le dictionnaire, mais le recensement des équivalences potentielles est tout simplement sans objet par rapport à la nature du dictionnaire, répertoire de signes et non de significations. Sous forme de boutade apparemment simpliste, Wittgenstein avait bien mis l'accent sur la stérilité de la recherche du sens hors contexte.

Dans une autre perspective, les recherches sur la traduction assistée par ordinateur viennent confirmer cette remarque de bon sens.

Dans le cadre d'une étude entreprise par le CELTA (Attali et al. 1992) sur le transfert lexical automatisé, les auteurs ont constaté que, en traduction automatique, cette opération consiste à 'remplacer du signifiant (source) par du signifiant (cible), [que] le choix entre les multiples équivalents cibles candidats à la traduction est le plus souvent dicté par des usages dépendant du contexte d'emploi. [...] Les termes monosémiques euxmêmes requièrent des traductions variables selon l'environnement immédiat'. Soulignons l'approche 'révolutionnaire' de cette conception du transfert lexical. Le projet est la réalisation d'un 'dictionnaire contextuel interactif, et l'angle d'étude, la TAO, vise à 
élucider les opérations d'exploration sémantico-contextuelle inconscientes effectuées par le traducteur, et que le concepteur du programme de TAO doit reconstituer afin de formaliser le méta-outil de traduction. Il s'agit de rechercher les 'régularités sémanticocognitives que les opérations de traduction peuvent contribuer à déceler'. À partir d'un corpus de trois millions et demi d'occurrences traduites (destinées à étudier cinq verbes), les auteurs ont réussi à définir les 'descripteurs sémantiques' qui ne sont plus établis a priori mais extraits de l'univers cognitif et culturel.

Le principal intérêt de cette démarche, c'est que l'unité lexicale traduite, l'équivalence, est enfin étudiée dans le contexte du discours; elle n'est plus simplement le résultat de l'activité intuitive, subjective, partiale, partielle bref empirique du lexicographe.

Son autre caractéristique, c'est que dès le départ, la question des équivalences est abordée soins un autre angle. S'il s'est bien agi, dans un premier temps, de recenser toutes les équivalences réalisées d'un terme de la langue source, l'étape suivante a été de chercher à faire la synthèse des 'descripteurs sémantiques' au lieu de multiplier les équivalences. En d'autres termes, c'est la démarche inverse à celles adoptées pour les dictionnaires bilingues: l'objectif n'est plus de rendre la variété des traductions possibles, mais de déterminer ce qu'elles ont en commun et ce qui explique qu'elles peuvent toutes apparaitre.

\section{De l'extension à l'intention : la notion}

L'étude de la fonction des équivalences dans un dictionnaire bilingue soulève en définitive des questions fondamentales qui dépassent la Iexicographie en tant que telle et dont elle est le révélateur. Car, de proche en proche, on a été amené à s'interroger sur la pertinence des subdivisions sémantiques (homo/polysémie), sur la nature de la définition, sur la nature du signe et sa fonction véritable dans un dictionnaire bilingue conçu comme aide au traducteur.

On a également montré que la multiplication des équivalences ne pouvait compenser les manques constitutifs: le travail en "extension» ( $\mathrm{du}$ sens) n'est pas une approche satisfaisante.

Les recherches évoquées ici sur la définition, sur la sémantique du prototype, sur la traduction automatique, ont finalement toutes pour caractéristiques le travail en intension (par opposition à I'extension). La signification du mot doit être élucidée par la détermination de son "sens basique », ou bien de ses "descripteurs sémantiques", et non plus par l'intermédiaire d'un signe.

Puisque le mot - en soi - ne renvoie pas à un sens (sinon comment expliquer qu'à un mot dans la langue source peuvent correspondre plusieurs mots dans la langue cible), c'est qu'en réalité, il renvoie à un «domaine notionnel, c'est-à-dire à un ensemble de virtualités » (Culioli 1990 : 86). Le terme « ensemble " ('union des parties dans un tout) étant a prendre au sens plein, chacune des virtualités concourant au recouvrement total du domaine notionnel.

60 Il en résulte alors que le mot est à considérer comme la réalisation ponctuelle d'une notion produit dans une situation d'énonciation donnée. Les équivalences du dictionnaire bilingue sont donc des réalisations ponctuelles du mot de la langue source. 
61 On perçoit plus clairement pourquoi le dictionnaire bilingue, approche descriptive du lexique, ne peut, sous sa forme actuelle, rendre compte de la subjectivité (virtuellement infinie) des situations d'énonciation. Le conflit apparaît donc comme insoluble entre l'objectivité désirée du dictionnaire et la subjectivité essentielle, au sens philosophique, de l'activité de langage.

Il n'en reste pas moins que nous avons besoin de dictionnaires bilingues et c'est peut-être en changeant d'objectif qu'il sera permis de concilier l'inconciliable : ne plus chercher à mettre en équivalence le mot, mais son domaine notionnel. En possession de ces éléments, l'utilisateur sera alors en mesure de « re-créer du sens » (Culioli 1990 : 86), qui est le propre de l'activité de langage.

\section{BIBLIOGRAPHIE}

Attali, A., G. Bourquin, M.C. Bourquin-Launay et al. 1992. « Aide au transfert lexical dans une perspective de TAO : expérimentation sur un lexique non terminologique ». Meta 37/4, 770-790. Benveniste, E. 1974. Problèmes de linguistique générale. vol. 2. Paris : Gallimard.

Collin, P. \& M. Schuwer. 1992. Dictionnaire de l'environnement et de l'écologie, anglais-français, français-anglais. Paris : Larousse.

Culioli, A. 1990. Pour une linguistique de l'énonciation, t. 1, Opérations et représentations. Gap : Ophrys. Harrap's Shorter. Dictionnaire anglais-français. 1991. Paris : Harrap.

Jakobson, R. 1963. Essais de linguistique générale. t.1, Les fondations du langage. Paris : Éd. de Minuit.

Kleiber, G. 1990. La sémantique du prototype. Paris : Presses Universitaires de France.

Lyons, J. 1968. Introduction to Theoretical Linguistics. Cambridge : Cambridge University Press.

Rey, A. 1990. « Polysémie de la définition ». In Centre d'études du lexique (dir.), La définition.

Paris : Larousse.

Robert \& Collins. Dictionnaire français-anglais, anglais-français. 1987. Paris : Le Robert.

Schuwer, Martine. 1993. «L'épreuve de vérité du retournement informatique ». ASp 1, 531-546.

\section{NOTES}

1. Hormis Mr Littré, dont on vient de publier l'ouvrage écrit en 1912, Comment j'ai écrit mon dictionnaire (référence non disponible).

2. Afin de faire clairement apparaître les sous-catégories, on a écarté tous les exemples qui illustrent l'emploi.

3. Ce paragraphe reprend des remarques essentielles de Rey (1990).

4. Wittgenstein, L. 1953. Philosophical Investigations, cité par J. Lyons (1968).

5. Cette argumentation est inspirée de E. Benveniste (1974 : vol. 2, chap. 15) 


\section{RÉSUMÉS}

Dans un dictionnaire bilingue, on conçoit bien l'objectif explicite du lexicographe qui propose, pour un terme, plusieurs équivalences dans la langue cible. Il rend ainsi compte de la nonisomorphie bien établie des champs sémantiques. Mais au-delà de ces informations, quelle fonction ces équivalences jouent-elles pour le lecteur?

In a bilingual dictionary, the lexicographer's goal is clearly to offer several possible equivalences in the target language for any term. In this way, he/she reflects the well-known nonisomorphism of semantic fields. Other than this information, however, what function do these equivalences have for the reader?

\section{INDEX}

Mots-clés : dictionnaire, lexicographie

Keywords : dictionary, lexicography

\section{AUTEUR}

\section{MARTINE SCHUWER}

Université de Haute Bretagne, Cerlico-Rennes. Martine.Schuwer@uhb.fr 